ORIGINAL ARTICLE

\title{
Indicators for preventable drug related morbidity: application in primary care
}

\author{
C J Morris, S Rodgers, V S Hammersley, A J Avery, J A Cantrill
}

See editorial commentary, p 170

Qual Saf Health Care 2004;13:181-185. doi: 10.1136/qshc.2003.008334

See end of article for authors' affiliations

.....................

Correspondence to: Dr C J Morris, The Drug Usage and Pharmacy Practice Group, School of Pharmacy and Pharmaceutical Sciences, University of Manchester, Manchester M13 9PL, UK; caroline.j.morris@ man.ac.uk

Accepted for publication 19 December 2003
Aim: To apply in practice a series of validated indicators for preventable drug related morbidity (PDRM). Design: A pilot study to identify retrospectively potential PDRM events over a 2 year 3 month time frame using the MIQUEST computer software program.

Subjects and setting: The electronic patient record of all patients aged 18 years and over in nine English general practices.

Outcome measures: The number of potential PDRM events identified, as defined by the indicators.

Results: Five hundred and seven potential PDRM events were identified from 49658 electronic patient records, giving an overall incidence of $1.0 \%$. A small number of the indicators $(n=4)$ accounted for approximately $60 \%$ of the events, while for many indicators few events were identified. The most common events related to the use of non-steroidal anti-inflammatory drugs in patients with congestive heart failure or hypertension, lack of monitoring in patients prescribed angiotensin converting enzyme inhibitors, and the use of hypnotic-anxiolytic agents.

Conclusions: A small number of indicators contributed to the majority of the PDRM events. Interrogation of electronic patient records in primary care using computerised queries shows potential for detecting PDRM.
$\mathrm{R}$ ecent reports from the US ${ }^{1}$ and the $\mathrm{UK}^{2}$ have raised the profile of the problem of drug related morbidity. Furthermore, drug related problems have been identified in a systematic review ${ }^{3}$ as a frequent cause of hospital admissions. This review identified, from 15 studies conducted worldwide, the median drug related hospital admission rate to be $7.1 \%$. In more than $50 \%$ of cases these admissions were judged to have been preventable. In a recent UK study ${ }^{4} 6.5 \%$ of admissions to a hospital medical admissions unit were considered to be drug related, with $67 \%$ judged to have been preventable. Preventable admissions were identified as being mainly due to problems with prescribing and monitoring of drug treatment.

The adverse clinical outcomes of drug related morbidity are potentially substantial, while the economic impact in ambulatory care patients in the US has been estimated to cost $\$ 177$ billion each year. ${ }^{5}$ Work in the area of preventable drug related morbidity (PDRM) has been primarily focused on secondary care in North America. No work has been undertaken in English primary care. This is surprising since the management of medicines in primary care is likely to make a

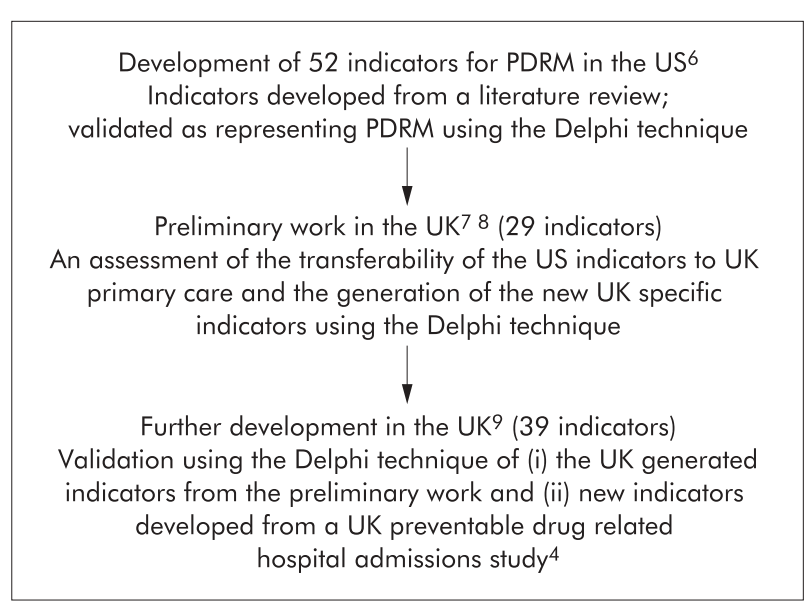

Figure 1 Development of PDRM indicators. significant contribution to the majority of preventable drug related hospital admissions. To develop effective strategies to reduce drug related morbidity and prevent drug related admissions, we need to be able to identify potential PDRM in primary care. The use of quality indicators representing PDRM is one possible way of doing so, thereby improving the safety and quality of health care. In the US, MacKinnon and Hepler $^{6}$ developed a series of PDRM indicators. Taking their work as a starting point, two of the authors (CJM and JAC) have already undertaken a substantial volume of work to produce validated indicators for PDRM in the UK. ${ }^{7-9} \mathrm{~A}$ summary of the indicator development is shown in fig 1 .

Building on a feasibility study, ${ }^{10}$ the work presented in this paper aimed to apply these indicators in three English primary care trusts (PCTs). The role and place of PCTs in the English NHS ${ }^{11}$ is shown in fig 2.

In this pilot study our objective was to apply the indicators retrospectively in computerised general practices to identify potential cases of PDRM. By doing so, we aimed to identify the most common PDRM events in these study practices.

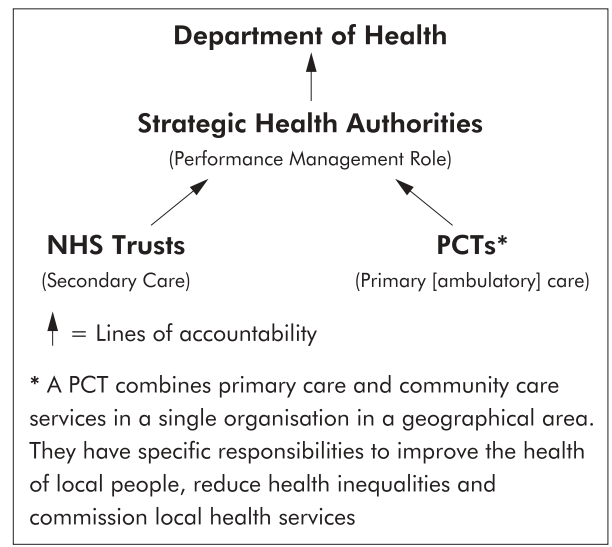

Figure 2 The place of primary care trusts (PCTs) in the NHS." 
Write queries to interrogate the EPR using the MIQUEST software

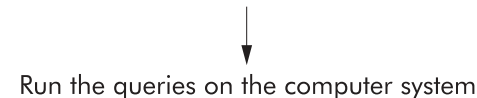
at each GP practice

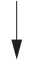

Extract the query reports from the GP computer system

Aggregate the data from all of the practices

Figure 3 Application of the indicators using MIQUEST software. $E P R=$ electronic patient record.

\section{METHODS}

Practices

Practices from three PCTs in the north west and east Midlands areas of England were eligible for inclusion in the study if they fulfilled the following inclusion criteria:

- Member of either the Trent Focus Collaborative Research Network or the Primary Care Information Services (PRIMIS) network.

- Running the EMIS 5 byte operating system.

- MIQUeST (Morbidity Information Query and Export Syntax) software installed.

- Electronically laboratory linked.

Inclusion was restricted to members of the specified networks because these organisations place an emphasis on the quality of the electronic patient record data.

\section{Applying the indicators in practice}

The number of potential PDRM events (as defined by the indicators) was assessed retrospectively by searching the electronic patient record of all patients aged 18 years and over in each practice over a 2 year 3 month time frame (1 November 1999 to 31 January 2002). The computer searches were run in each practice by a member of the research team (CJM, SR or VSH) between July and November 2002. This was achieved using the MIQUEST computer software program. The steps involved in this process are shown in fig 3.

\section{PDRM indicators}

A series of indicators for PDRM were applied in the GP practices. A summary of their derivation is shown in fig 1 . From this previous work we had validated 39 PDRM indicators suitable for application in primary care..$^{7-9}$ However, only 29 indicators were successfully applied in this

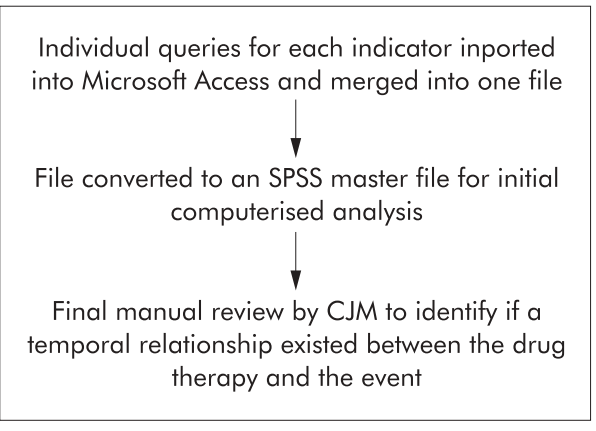

Figure 4 The analysis process.

\section{Box 1 Reasons for exclusion of 10 indicators}

- Data not in electronic patient record $(n=6)$, for example:

Outcome: hospital admission due to an acute exacerbation of asthma or chronic obstructive airways disease (COAD).

Pattern of care: dispensing and issuing a prescription by a pharmacist for $\beta$ blocker eye drops to a patient with a known history of asthma or COAD without advising them to contact their GP in the event of any deterioration of their respiratory symptoms.

- Too complex to be written as a MIQUEST query $(n=3)$, for example:

Outcome: osteoporosis or broken bone.

Pattern of care: use of long term steroids at a dose equivalent to $\geqslant 7.5 \mathrm{mg}$ prednisolone per day without osteoporosis prophylaxis.

- Technical error in query: miscoding a diagnosis $(n=1)$

study. The reasons for the exclusion of 10 indicators are shown in box 1 .

The indicators encompass a variety of different clinical situations and all take the form of an adverse therapeutic outcome (the PDRM event) resulting from an associated pattern of patient care.

\section{Data analysis}

Up to six individual queries were required to collect the relevant data for each indicator, thus considerable data manipulation was required after data extraction. This process was automated as far as possible and is shown in fig 4 .

Completing this process identified the number of potential PDRM events for each indicator and therefore the most commonly occurring events in the study practices.

The study was approved by the local research ethics committee in each locality and the University ethical committee.

\section{RESULTS}

Fifteen practices were approached, 12 of which agreed to participate in the study. However, data from only nine practices were used as two were not laboratory linked and one did not have functioning software. In addition, two of the practices had linked computer support and were therefore treated as a single practice for the purposes of this study.

Table 1 Incidence of preventable drug related morbidity (PDRM) events in individual practices

\begin{tabular}{llll}
$\begin{array}{l}\text { Practice } \\
\text { no }\end{array}$ & $\begin{array}{l}\text { Records reviewed } \\
(\mathbf{n = 4 9 6 5 )}\end{array}$ & $\begin{array}{l}\text { PDRM events } \\
\text { (n=507) }\end{array}$ & $\begin{array}{l}\text { PDRM incidence } \\
(\%)\end{array}$ \\
\hline 2 & 7095 & 132 & 1.9 \\
1 & 5153 & 77 & 1.5 \\
6 & 3026 & 37 & 1.2 \\
3 & 6053 & 60 & 1.0 \\
5 & 6014 & 53 & 0.9 \\
4 & 6031 & 42 & 0.7 \\
$7 *$ & 12945 & 97 & 0.7 \\
8 & 3341 & 9 & 0.3 \\
& $*$
\end{tabular}


Table 2 Number of potential preventable drug related morbidity (PDRM) events by indicator and range of number of events at individual practice level

\begin{tabular}{l} 
Indicator details \\
\hline Outcome: GP practice or hospital contact due to CHF and/or fluid overload \\
Pattern of care: Use of an oral/topical NSAID for 3 months or more in a \\
patient with hypertension and/or CHF \\
Outcome: Raised serum creatinine ( $\geqslant 150 \mu$ mol/I) \\
Pattern of care: Use of an ACE inhibitor without monitoring the creatinine \\
level before starting treatment, within 6 weeks of commencement, and at
\end{tabular}

least annually thereafter

Outcome: Hyperkalaemia (potassium level $\geqslant 5.5 \mathrm{mmol} / \mathrm{l}$ )

Pattern of care: Use of an ACE inhibitor without monitoring the potassium

level before starting treatment, within 6 weeks of commencement, and

at least annually thereafter

Outcome: Fall or broken bone

Pattern of care: Use of a long half life hypnotic-anxiolytic

61

Outcome: A second MI

Pattern of care: In the absence of any contraindication, failing to prescribe a $\beta$ blocker in a patient with a history of an $\mathrm{Ml}$

Outcome: Dyspepsia or upper GI bleed, GI perforation, GI ulcer or anaemia Pattern of care: Use of an oral/topical NSAID for 1 week or more in

a patient with a history of peptic ulcers or GI bleeding

Outcome: GP contact or hospital admission due to worsening symptoms of $\mathrm{CHF}$

Pattern of care: In the absence of any contraindication, failing to prescribe an ACE inhibitor to a patient with known CHF

Outcome: Blood dyscrasias

Pattern of care: Use of carbamazepine without a full blood count before treatment initiated and periodically during treatment

Outcome: GP practice or hospital contact due to asthma symptoms Pattern of care: Use of an inhaled short acting bronchodilator more than once daily or at night in an asthmatic patient with no regular inhaled "preventer" therapy (corticosteroid or cromoglycate or nedocromil)

Outcome: GP or hospital contact due to an exacerbation of asthma or COAD Pattern of care: Use of $\beta$ blocker in a patient with asthma or COAD

Outcome: A minor or major haemorrhagic event

Pattern of care: Use of warfarin without monitoring the INR before initiation of treatment, on alternate days in the early days of treatment, then at longer intervals, then at least every 3 months thereafter

Outcome: Hypokalaemia (potassium level $\leqslant 3.0 \mathrm{mmol} / \mathrm{l}$ )

Pattern of care: Use of a potassium wasting diuretic without concurrent use of a potassium supplement or concurrent use of a potassium sparing diuretic or monitoring the potassium level at least annually

Outcome: A second MI

Pattern of care: In the absence of any contraindication, failing to prescribe aspirin in a patient with a history of $\mathrm{MI}$

Outcome: Oral thrush/dysphonia

Pattern of care: Use of an inhaled steroid by high dose metered dose inhaler without usage of a spacer device

Outcome: GP practice or hospital contact due to hyperthyroidism Pattern of care: Use of a thyroid agent without monitoring the $\mathrm{T}_{4}$ or thyroid stimulating hormone within 6 weeks of initiation of treatment and at least every 12 months thereafter 
Table 2 Continued

\begin{tabular}{l} 
Indicator details \\
\hline Outcome: A minor or major haemorrhagic event \\
Pattern of care: Concurrent use of warfarin and an oral/topical NSAID \\
without monitoring the INR within 10 days
\end{tabular}

Outcome: A minor or major haemorrhagic event

Pattern of care: Concurrent use of warfarin and an antibiotic without

monitoring the INR within 5 days

Outcome: Acute urinary retention

Pattern of care: Use of an anticholinergic agent in a patient with a history

or current diagnosis of benign prostatic hypertrophy

Outcome: Serum transaminase concentrations elevated to three times the upper limit of the reference range or clinical jaundice

Pattern of care: Use of a statin without monitoring the liver function before

starting treatment, within 3 months of commencement and then at

6 monthly intervals thereafter

Outcome: GP or hospital contact due to a deterioration in symptoms, or an acute exacerbation, of asthma or COAD

Pattern of care: Prescribing $\beta$ blocker eye drops to a patient with a history of

asthma or COAD

Outcome: Drowsiness or confusion or arrhythmias or delirium or hallucinations

Pattern of care: Continued use of a previously established dose of digoxin without assessing the digoxin level in a patient presenting with any of the following symptoms: anorexia, nausea and vomiting, diarrhoea, visual disturbances, fatigue

Outcome: Dyspepsia or upper GI bleed, GI perforation, Gl ulcer or anaemia Pattern of care: Use of an oral corticosteroid for at least 3 months in a patient with a history or current diagnosis of peptic ulcers and/or GI bleeding

Outcome: GP practice or hospital contact due to $\mathrm{CHF}$ and/or heart block Pattern of care: Use of digoxin in a patient with CHF, with heart block or advanced bradycardia

Outcome: Worsening of Parkinson's disease symptoms e.g. attacks of rigidity or tremor

Pattern of care: Use of metoclopramide in a patient with a history

of Parkinson's disease

5

3

Outcome: Acute urinary retention

Pattern of care: Use of imipramine in a patient with a history or current

diagnosis of bladder atony resulting from diabetes

Outcome: Anorexia or nausea and vomiting or diarrhoea or visual disturbances or fatigue or drowsiness or confusion or arrhythmias or delirium or hallucinations

Pattern of care: Addition of amiodarone to the treatment of a patient already prescribed digoxin without reducing the digoxin dosage by initially one

third to one half and subsequent monitoring of the digoxin level

Outcome: Hyperkalaemia (potassium level $\geqslant 5.5 \mathrm{mmol} / \mathrm{l}$ )

Pattern of care: Concurrent use of an ACE inhibitor and either a potassium sparing diuretic or a potassium supplement without monitoring the potassium level at least annually

Outcome: A minor or major haemorrhagic event

Pattern of care: Addition of amiodarone to the treatment of a patient

already prescribed warfarin without reducing the warfarin dose and closely monitoring the INR

Outcome: Hospital admission due to loss of seizure control.

Pattern of care: Continued use of a previously established dose of phenytoin

without assessing phenytoin level in a patient experiencing an altered seizure pattern

$\mathrm{CHF}=$ congestive heart failure; $\mathrm{NSAID}=$ non-steroidal anti = inflammatory drug; $\mathrm{ACE}=$ angiotensin converting enzyme; $\mathrm{Ml}=$ myocardial infarction; $\mathrm{COAD}=$ chronic obstructive airways disease; $I N R$ = international normalised ratio; $\mathrm{Gl}=$ gastrointestinal. 


\section{Key messages}

- A potential PDRM event was identified in $1.0 \%$ of electronic patient records.

- Four indicators accounted for approximately $60 \%$ of these events.

- The most commonly occurring events related to the prescribing of non-steroidal anti-inflammatory drugs and hypnotic-anxiolytics and the monitoring of angiotensin converting enzyme inhibitors.

The number of GP partners ranged from 1 to 7 . Five practices held GP training status and they were located in urban $(n=3)$, suburban $(n=3)$, and rural areas $(n=2)$.

\section{PDRM events}

A total of 49658 electronic patient records were identified for patients aged 18 years and over which were eligible for interrogation by the MIQUEST queries. In all, 507 potential PDRM events were identified, giving an overall incidence of PDRM of $1.0 \%$. The incidence of PDRM at the individual practice level is shown in table 1 . Table 2 shows the total number of events identified for each indicator and the range of numbers of events for each indicator in individual practices. It can be seen from table 2 that four indicators accounted for the majority of the events $(302 / 507,59.6 \%)$. These related to the use of nonsteroidal anti-inflammatory drugs in patients with congestive heart failure or hypertension, lack of monitoring of potassium and creatinine in patients prescribed angiotensin converting enzyme (ACE) inhibitors, and the use of hypnotic-anxiolytic agents. No events were identified for five indicators, 1-10 for 11 indicators; 11-20 for five indicators, and 21-40 for four indicators.

\section{DISCUSSION}

This pilot study has shown that a substantial number of potential PDRM events occur in English primary care. The computerised queries were able to interrogate GP systems, resulting in the identification of potential PDRM events in one in 100 electronic patient records.

Identification of potential PDRM events enables us to consider strategies that are likely to have the greatest impact on reduction of future PDRM. This in turn will benefit patients by improving the safety and the quality of health care and also have a positive impact on healthcare resource utilisation.

It is notable that most of the indicators contributed to very few events, while four (the prescribing of NSAIDs and hypnotic-anxiolytics and the monitoring of ACE inhibitors) contributed to approximately $60 \%$ of the PDRM events. However, the clinical implications for individual patients may be great, irrespective of the number of events identified by each indicator. To date, limited research has been undertaken on PDRM. As this work took place in the North American healthcare system, ${ }^{6}{ }^{12}{ }^{13}$ only cautious comparisons can be made. Nevertheless, despite the fact that MacKinnon and Hepler $^{12}$ applied PDRM indicators within a managed care organisation using the database of a hospital based healthcare plan in Florida, a similar pattern emerged. A small number of indicators accounted for the majority of the PDRM events. Furthermore, some similarities existed in the clinical issues that contributed to the seven most frequently occurring PDRM events, ${ }^{12}$ with NSAIDs, ACE inhibitors, and failing to prescribe a $\beta$ blocker after myocardial infarction appearing in both data sets.

However, when interpreting the study results a number of factors need to be considered. Although a temporal relationship was identified between drug therapy and the PDRM event, it does not necessarily mean that the drug was the direct or only cause of that event. Furthermore, the quality of our data is dependent upon the quality of the data present on the practice system. Although prescribing data are generally of better quality than diagnostic or lifestyle data on primary care computer systems, ${ }^{14}$ we accept that the quality of data recording in the practices was beyond our control. For this reason, the PDRM incidence data should be interpreted cautiously. At present it would be inappropriate to use these data to draw comparisons between practices but, with current moves to improve data recording in the UK and the implications of the new GP contract, ${ }^{15}$ computerised data from general practices are likely to be more reliable in the future and appropriate comparisons may then prove useful. Despite some practical difficulties, electronic patient records have considerable potential in the detection of PDRM.

We have shown that a substantial number of potential PDRM events are occurring in primary care. However, because of the problems of assigning direct causality of the drug to the event, rather than aiming to collect prevalence data our ultimate purpose is to use the indicator data to generate discussion in facilitated multidisciplinary discussion forums attended by key practice personnel (GPs, nurses, pharmacists and the practice manager). These will use the key principles of root cause analysis (prioritisation, root cause identification and root cause elimination). ${ }^{16}$ The outcome of these meetings will form the focus of a subsequent paper describing how the overall process can facilitate improvements in medicines management and ultimately the quality of patient care.

\section{ACKNOWLEDGEMENTS}

We would like to thank the staff at all the GP practices for their help and support with this study.

\section{Authors' affiliations}

C J Morris, J A Cantrill, School of Pharmacy and Pharmaceutical Sciences, University of Manchester, Manchester, UK

S Rodgers, V S Hammersley, A J Avery, Division of General Practice, University of Nottingham, Nottingham, UK

\section{REFERENCES}

1 Kohn LT, Corrigan JM, Donaldson MS. To err is human: building a safer health system. Washington, DC: Institute of Medicine, 1999.

2 Department of Health. An organization with a memory. London: Department of Health, 2000.

3 Winterstein AG, Sauer BC, Hepler CD, et al. Preventable drug-related hospital admissions. Ann Pharmacother 2002;36:1238-48.

4 Howard RL, Avery AJ, Howard PD, et al. Investigation into the reasons for preventable drug related admissions to a medical admissions unit: observational study. Qual Saf Health Care 2003;12:280-5.

5 Ernst FR, Grizzle AJ. Drug-related morbidity and mortality: updating the costof-illness model. J Am Pharm Assoc 2001;41:192-9.

6 MacKinnon NJ, Hepler CD. Preventable drug-related morbidity in older adults. 1. Indicator development. J Man Care Pharm 2002;8:365-71.

7 Morris CJ, Cantrill JA, Hepler CD, et al. Preventing drug-related morbidity: determining valid indicators. Int J Qual Health Care 2002;14:183-98.

8 Morris C, Cantrill J. Preventable drug-related morbidity indicators in the US and UK. J Man Care Pharm 2002;8:372-7.

9 Morris CJ, Cantrill JA. Preventing drug-related morbidity: the development of quality indicators. J Clin Pharm Ther 2003;28:295-305

10 Morris CJ, Cantrill JA, Bate JR. Indicators for preventable drug-related morbidity: facilitating improvements in patient care. Pharmacoepidemiol Drug Saf 2002;11(Suppl 1):S23.

11 Department of Health. The new NHS. www.doh.gov.uk/about/newsnhs.htm (accessed 21 July 2003).

12 MacKinnon NJ, Hepler CD. Indicators of preventable drug-related morbidity in older adults 2 . Use within a managed care organization. J Man Care Pharm 2003;9:134-41.

13 Robertson HA, MacKinnon NJ. Development of a list of consensus-approved clinical indicators of preventable drug-related morbidity in older adults. Clin Ther 2002;24:1595-613.

14 Thiru K, Hassey A, Sullivan F. Systematic review of scope and quality of electronic patient record data in primary care. BMJ 2003;326:1070-4

15 Shekelle P. New contract for general practitioners. BMJ 2003:326:457-8.

16 Andersen B, Fagerhaug T. Root cause analysis: simplified tools and techniques. Milwaukee, WI: ASQ Quality Press, 2000. 